\title{
HAMARTOMA COMBINADO DE RETINA Y EPITELIO PIGMENTARIO RETINIANO. DIAGNÓSTICO MEDIANTE TOMOGRAFÍA DE COHERENCIA ÓPTICA Y ANGIOFLUORESCEINGRAFÍA
}

\author{
COMBINED HAMARTOMA OF THE RETINA AND RETINAL \\ PIGMENT EPITHELIUM DIAGNOSED BY RETINAL \\ ANGIOGRAPHY AND OPTICAL COHERENCE TOMOGRAPHY
}

\author{
PÉREZ-ÁLVAREZ MJ ${ }^{1}$, ALEJANDRE-ALBA N¹, GARCÍA-SÁNCHEZ J²
}

\section{RESUMEN}

Caso clínico: Se presenta el caso de una paciente de 35 años, que desarrolló un hamartoma combinado de retina y epitelio pigmentado retiniano. El diagnóstico se determinó en base a los característicos hallazgos funduscópicos: hiperplasia del epitelio pigmentado retiniano, tortuosidad vascular y membrana epirretiniana.

Discusión: La tomografía de coherencia óptica y la angiofluoresceingrafía permiten descartar melanoma coroideo o retinoblastoma. Es importante realizar un seguimiento periódico por la posibilidad de afectación visual por membrana neovascular coroidea, membrana epirretiniana o hemorragia vítrea.

Palabras clave: Hamartoma combinado de retina y epitelio pigmentado retiniano, tomografía de coherencia óptica.

\section{ABSTRACT}

Clinical case: This report is based on the case of a 35-year-old woman who developed a combined hamartoma of the retina and retinal pigment epithelium in her right eye. The diagnosis was determined based on the fundus examination: hyperplasia of the retinal pigment epithelium cells, tortuosity of the vessels and epiretinal membrane.

Discussion: Optical coherence tomography and retinal angiography are important to rule out malignant melanoma of the choroid and retinoblastoma. Regular follow-up is essential because reduction in visual acuity can be related to an epiretinal membrane, neovascular membrane or vitreous hemorrhage (Arch Soc Esp Oftalmol 2008; 83: 193-196).

Key words: Hamartoma of the retina and retinal pigment epithelium, Optical coherence tomography.

\footnotetext{
Recibido: 15/9/06. Aceptado: 18/2/08.

Hospital Clínico San Carlos. Universidad Complutense de Madrid. Madrid. España.

1 Licenciado en Medicina.

2 Doctor en Medicina.

Correspondencia:

María Jesús Pérez Álvarez

Paseo de los Pontones, 5, 4. ${ }^{\circ}$ Dcha.

28005 Madrid

España

E-mail: mjperezalv@hotmail.com
} 


\section{INTRODUCCIÓN}

Los hamartomas combinados de retina y epitelio pigmentario retiniano (CHRRPE) son raros tumores benignos, caracterizados por la proliferación del epitelio pigmentario retiniano (EPR) y tejido glial, generando gran distorsión papilar y retiniana. Se aprecia una lesión solitaria unilateral sobrelevada, hiperpigmentada, acompañada de tortuosidad vascular y membrana epirretiniana (MER).

\section{CASO CLÍNICO}

Se presenta el caso de una mujer de 35 años que acudió por miodesopsias en el ojo derecho (OD). En la exploración se evidenció: Agudeza visual (AV) en ambos ojos corregida: 1. No se apreciaron nódulos iridianos en la biomicroscopía anterior. Tonometría: normal. Funduscopía: OD: Lesión peripapilar nasal, sobrelevada e hiperpigmentada, con vasos tortuosos telangiectásicos y MER, y atrofia coriorretiniana peripapilar subyacente a la lesión (fig. 1). En la periferia se detectó una zona de tracción vítrea, posiblemente causa de los síntomas, que fue fotocoagulada con láser argón. El ojo izquierdo fue normal (fig. 2).

$\mathrm{La}$ angiofluoresceingrafía (AFG) mostró bloqueo de fluorescencia en tiempo precoces y vasos telangiectásicos con fuga de contraste y ligera tinción de la lesión en tiempos tardíos, no se objetivaron signos de neovascularización coroidea ni retiniana (fig. 3).

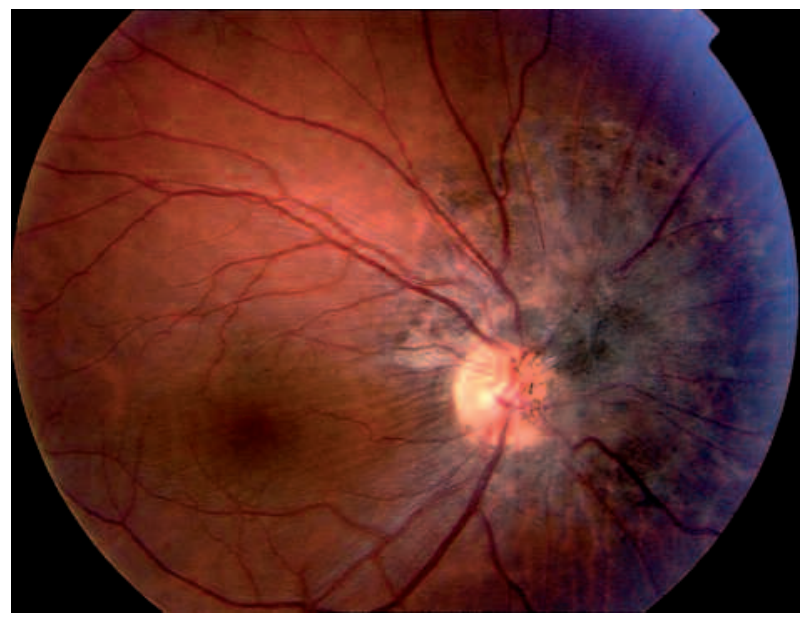

Fig. 1: Funduscopía del ojo derecho que muestra lesión característica sobrelevada pigmentada con vasos telangiectásicos.

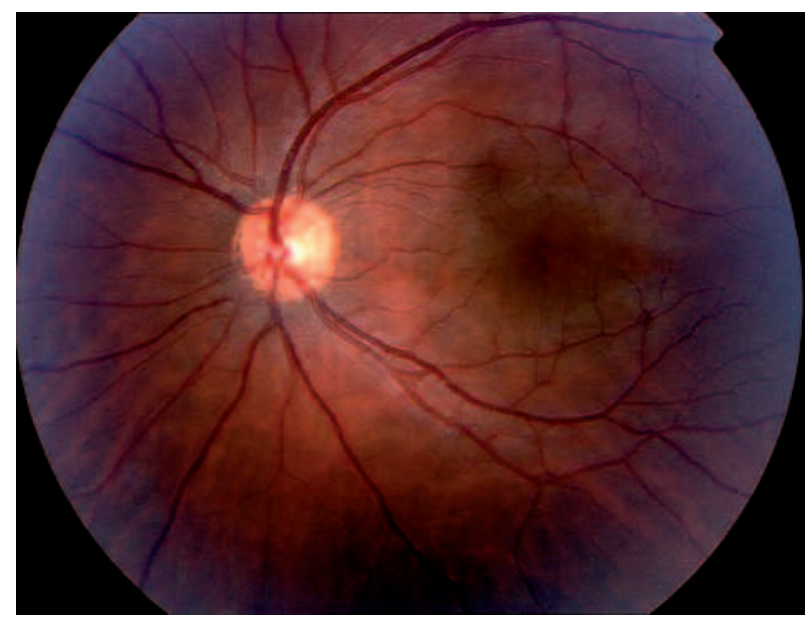

Fig. 2: Funduscopía del ojo izquierdo sin alteraciones.

En la ecografía ocular no se apreció elevación significativa de la lesión ni signos sugestivos de melanoma coroideo.

La tomografía de coherencia óptica (OCT) mostró una elevación retiniana hiperreflectante con sombra hiporreflectante subyacente e importante distorsión de toda la arquitectura retiniana dentro de los límites de las lesión, con clara MER (figs. 4 y 5).

En la perimetría computarizada Humphrey 24-2 la lesión generó un escotoma absoluto.

Ante la sospecha de hamartoma combinado de retina y epitelio pigmentario retiniano (CHRRPE) se remitió al servicio de medicina interna, para descartar enfermedad asociada.y se realizó una reso-

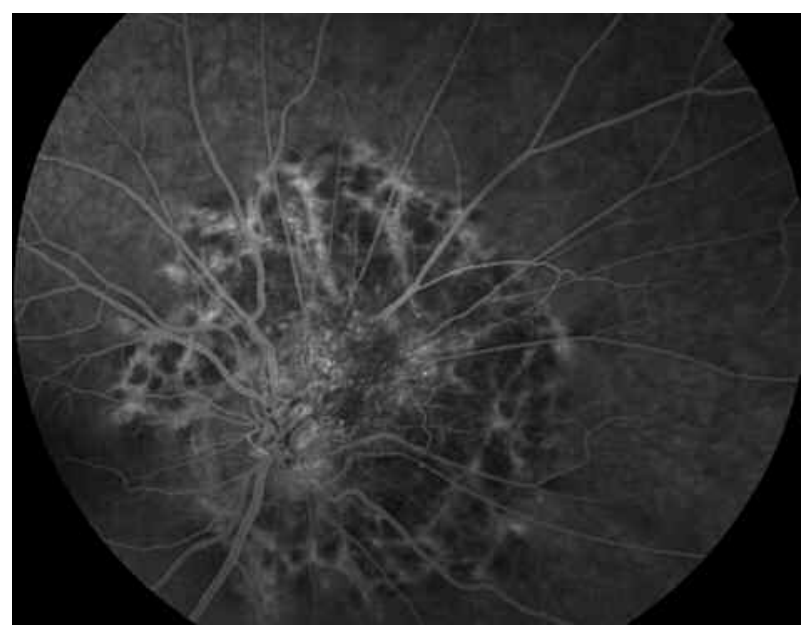

Fig. 3: Tiempos tardios donde se aprecia fuga desde dilataciones telangiectásicas y leve tinción de la lesión. 

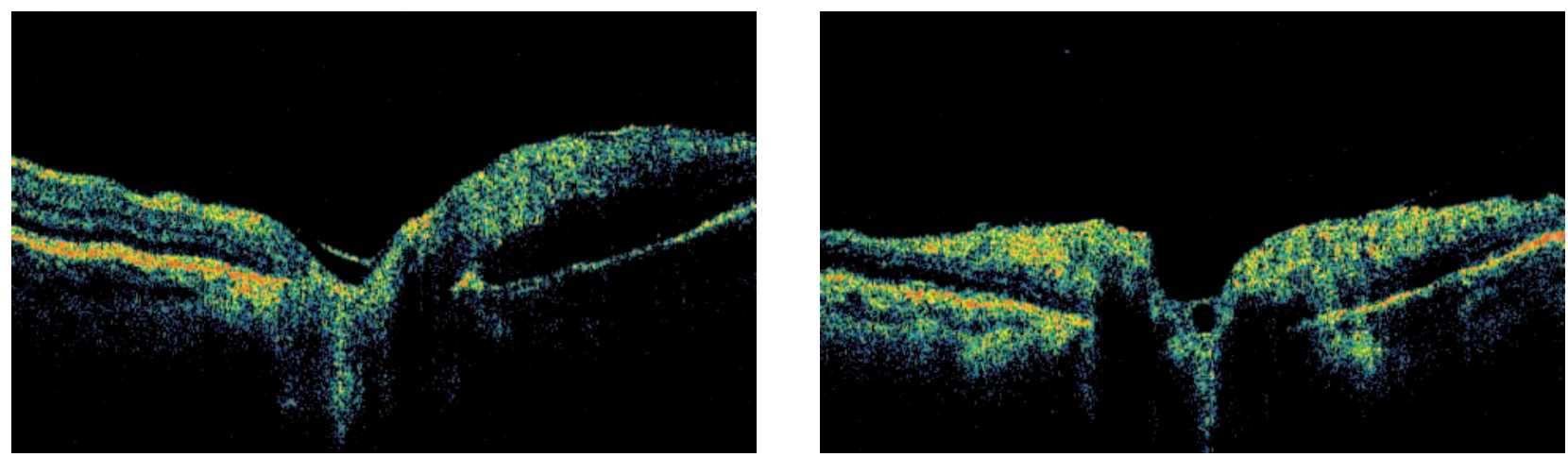

Fig. 4 y 5: Tomografía de coherencia óptica en la que se aprecia la distorsión de la arquitectura retiniana, lesión hiperreflectante sobrelevada con membrana prerretiniana.

nancia magnética nuclear (RMN) craneal y orbitaria.Todo el estudio sistémico resultó normal.

A los 6 meses de evolución la lesión permanece estable sin disminución de la AV ni aparición de metamorfopsias u otras complicaciones.

\section{DISCUSIÓN}

Un hamartoma constituye una proliferación de carácter benigno de células que se hallan normalmente en esa localización, por tanto el CHRRPE presenta malformación del EPR, retina neurosensorial, vascularización y vítreo adyacente, en grado variable. El $93 \%$ de los casos presentan tortuosidad vascular, hiperpigmentación el 87\%, lesión sobreelevada el $80 \%$, MER el $78 \%$. Habitualmente son lesiones únicas, unilaterales, localizadas próximas al nervio óptico $(76 \%)$, en la mácula $(17 \%)$ o periféricas $(7 \%)(1,2)$.

El $10 \%$ de los casos constituyen un hallazgo casual, siendo la clínica más frecuente la pérdida indolora de AV (60\%), otras manifestaciones pueden ser estrabismo (18\%), miodesopsias (5\%), leucocoria y dolor $(5 \%)$. La afectación directa de la fóvea, del haz papilomacular o del nervio óptico son las causas más frecuentes de disminución de AV. Aunque también puede deberse a la tracción causada por la MER, exudación intra o subretiniana a partir de los componentes vasculares de la lesión (2).

En la mayoría el curso es estacionario (2), sin embargo en ocasiones puede existir pérdida de visión tardía debida a :hemorragia vítrea (HV), membrana neovascular coroidea (MNVC), edema macular crónico, retinosquisis progresiva, agujero macular, desprendimiento de retina (DR) y neovas- cularización retiniana (1). El crecimiento de la lesión es excepcional.

El diagnóstico, como ya hemos apuntado, se basa en la funduscopía y se confirma mediante OCT y AFG $(2,3)$. La OCT es muy característica con gran distorsión de todas las estructuras, MER y tracción vitreorretiniana. Esta prueba puede constituir una herramienta para determinar la utilidad de la vitrectomía vía pars plana (VPP) con pelado de la MER en casos seleccionados (4).

La ecografía no siempre es precisa porque no suelen ser lesiones muy elevadas y no presentan patrón típico ultrasonográfico.

El diagnóstico diferencial debe realizarse con tumores coroideos y retinoblastoma $(2,3)$, para ello son útiles las pruebas ya referidas.

La mayoría no presenta manifestaciones sistémicas. Existen casos publicados con neurofibromatosis tipo 1 y 2 , aunque esta asociación no está bien establecida aún. También puede asociarse a hemangioma facial, incontinencia pigmento y esclerosis tuberosa Por ello son recomendables pruebas de neuroimagen (1).

La mayoría no precisa tratamiento. Es recomendable la oclusión para prevenir la ambliopía en niños. Las indicaciones de la VPP no están todavía bien establecidas con respecto al pelado de la MER Los casos descritos en la bibliografía presentan resultados visuales desiguales.Esta controversia es debida a que la lesión histológicamente es intrarretiniana, por tanto sólo se demuestra mejoría en aquellos casos en los que se objetive clara tracción vítrea (4). La VPP estaría indicada como tratamiento de las complicaciones (HV, DR) con resultados variables según los autores (1,5). Algunos autores describen buenos resultados visuales tras la cirugía 
submacular como tratamiento de la MNVC, debido a que éstas se localizan por encima del EPR (tipo 2 de Gass) (5).

Podemos concluir que, aunque se trata de tumores benignos debemos realizar un adecuado diagnóstico diferencial, mediante AFG y OCT, además de un seguimiento periódico debido al riesgo de pérdida de AV por las complicaciones asociadas, como MER, MNVC, HV y DR.

\section{BIBLIOGRAFÍA}

1. Helbig H, Niederberger H. Presumed combined hamartoma of the retina and retinal pigment epithelium with pre- retinal neovascularization. Am J Ophthalmol 2003; 136: 1157-1159.

2. Hamdi R, De Korvin H, Speeg-Schatz C, Szwarcberg J. Combined hamartoma of the retina and retinal pigment epithelium. Four case studies. J Fr Ophtalmol 2006; 29: e6.

3. Shields CL, Materin MA, Shields JA. Review of optical coherence tomography for intraocular tumors. Curr Opin Ophthalmol 2005; 16: 41-54.

4. Stallman JB. Visual improvement after pars plana vitrectomy and membrane peeling for vitreoretinal traction associated with combined hamartoma of the retina and retinal pigment epithelium. Retina 2002; 22: 101-104.

5. Inoue M, Noda K, Ishida S, Yamaguchi T, Nagai N, Shinoda K, et al. Successful treatment of subfoveal choroidal neovascularization associated with combined hamartoma of the retina and retinal pigment epithelium. Am J Ophthalmol 2004; 138: 155-156. 